EPJ Web of Conferences 37, 09005 (2012)

DOI: $10.1051 /$ epjconf/20123709005

(C) Owned by the authors, published by EDP Sciences, 2012

\title{
The measurement of Matveev-Muradyan-Tavkhelidze-Drell- Yan processes with SPD detector at NICA. Background studies.
}

\author{
Batozskaya V.S. ${ }^{\text {a }}$ \\ Joint Institute for Nuclear Research
}

\begin{abstract}
SPD NICA project is under preparation at second interaction point of the NICA collider. The purpose of this experiment is the study of the nucleon spin structure with high intensity polarized light nuclear beams. It is argued that the design of the collider can allow us to reach with proton beams a very high collision energy up to $\sqrt{s} \sim 26$ $\mathrm{GeV}$ with average luminosity up to $10^{30}-10^{31} \mathrm{~cm}^{-2} \mathrm{~s}^{-1}$. At the same time, the respective number for deuteron collisions is also quite considerable: at a collision energy per nucleon up to $\sqrt{s} \sim 12 \mathrm{GeV}$, the average luminosity reaches up to $10^{29}-10^{30} \mathrm{~cm}^{-2} \mathrm{~s}^{-1}$. It is of great importance that both proton and deuteron beams can be effectively polarized. The preliminary design of the SPD detector for spin physics studies is based on the requirements imposed by the MMT-DY and $J / \psi$ productions studies. The some sources of background to the MMT-DY process are the combinatorial background from decays $\left(\pi^{0}, \eta\right)$ and gamma contribution and the decays of charmed-mesons, which are studied now.
\end{abstract}

\section{Introduction}

The goal of the Spin Physics program at NICA is the study of the nucleon spin structure with high intensity polarized proton and deuteron beams in the collider mode. The collider will allow us to reach a very high collision proton energy up to $\sqrt{s} \sim 26 \mathrm{GeV}$ with a luminosity up to $10^{30}-10^{31} \mathrm{~cm}^{-2} \mathrm{~s}^{-1}$. For deuteron beams, the collision energy per nucleon up to $\sqrt{s} \sim 12 \mathrm{GeV}$ with an average luminosity up to $10^{29}-10^{30} \mathrm{~cm}^{-2} \mathrm{~s}^{-1}$. Both proton and deuteron beams can be effectively polarized. All these advantages give us unique possibilities to investigate at NICA the polarized phenomena which are of crucial importance for the solution of the nucleon spin problems - one of the main tasks of modern high energy physics

\section{The NICA project}

The purpose of the NICA project is the construction of a new accelerator facility at JINR (Dubna) that consists of a cryogenic heavy ion source, a source of polarized protons and deuterons, the "old" linac LU-20, a new heavy ion linear accelerator, a new Booster-synchrotron, the existing proton synchrotron (Nuclotron), upgraded to Nuclotron-M, two new superconducting storage rings of the collider, and a set of transfer channels (see fig. 1).

The object will have to provide: ion-ion $(\mathrm{Au})$ and ion-proton collisions in the energy range of 1 to $4.5 \mathrm{GeV} / \mathrm{u}$ at a luminosity up to $\mathrm{L} \sim 10^{27} \mathrm{~cm}^{-2} \mathrm{~s}^{-1}$ and collisions of polarized proton-proton (deuterondeuteron) beams in the energy range of 5 to $12.6 \mathrm{GeV}(2$ to $5.8 \mathrm{GeV} / \mathrm{u})$ at a luminosity $\mathrm{L}>10^{30}$ $\mathrm{cm}^{-2} \mathrm{~s}^{-1}$. Using Nuclotron-M and NICA facilities the following set of experiments is presently being

a e-mail: Varvara.Batozskaya@cern.ch

This is an Open Access article distributed under the terms of the Creative Commons Attribution License 2.0, which permits unrestricted use, distribution, and reproduction in any medium, provided the original work is properly cited. 


\section{EPJ Web of Conferences}

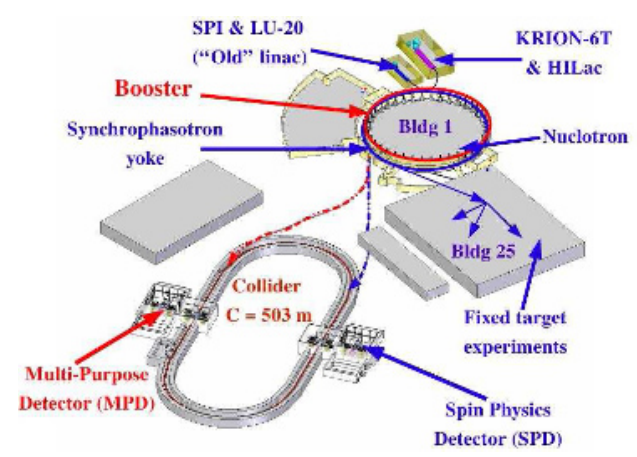

Fig. 1. Scheme of the Nuclotron-based Ion Collider fAcility (NICA).

planned: fixed target experiments, experiments with an internal target, collider experiments with two interaction points and respectively with two detectors. The first one is the Multi Purpose Detector (MPD), aiming at experimental studies of hot and dense strongly interacting QCD matter and the search for a possible manifestation of signs of the mixed phase and critical endpoint in heavy ion collisions. The second one will be used for the spin physics studies and is called Spin Physics Detector (SPD) [1].

\section{Spin Physics at NICA}

\subsection{Proposed measurements}

The parameters of the NICA collider allow us to execute the studies of:

- MMT-DY processes with longitudinally and transversely polarized $p$ and $d$ beams. Extraction of parton distribution functions (PDFs);

- PDFs from $J / \psi$ production processes;

- cross sections, helicity amplitudes and double spin asymmetries in elastic reactions;

- polarization effects in heavy ion collisions;

- and other questions.

\subsection{SPD detector}

The proposed view of the SPD detector is based on the requirements imposed by the MMT-DY and $J / \psi$ productions studies. These requirements are the following: almost $4 \pi$ geometry for secondary particles, precise vertex detector, precise tracking system, precise momentum measurement of secondary particles, good particle identfication capabilities $(\mu, \pi, p, e$, etc.). The preliminary design of the SPD is shown in fig. 2. The main parts of this scheme: toroid magnet system (TM), silicon detector (VC), drift chambers (DC), electromagnetic calorimeter (EMC), range system (RS) and trigger system (TS), which are described below.

The TM of the spectrometer provides a field free region around the interaction point and doesn't disturb the trajectories. The TM can consist of 8 superconducting coils, symmetrically placed around the beam axis.

Preliminary studies show that the use of superconducting coils allows us to reach an azimuthal detector acceptance in excess of $85 \%$, while the radius of the inner magnet volume can be about 0.3 $\mathrm{m}$ and the outer one about $0.7 \mathrm{~m}$, with $\int B d L \sim 1 \mathrm{Tm}$. Several layers of double-sided Silicon strips can provide a precise vertex reconstruction and tracking of the particles before they reach the magnet. The coordinate resolution of $150-200 \mu \mathrm{m}$ can be achieved with conventional DC. This can provide a momentum resolution of the order of 1-3\% over the kinematic range of the detector. The EMC will 


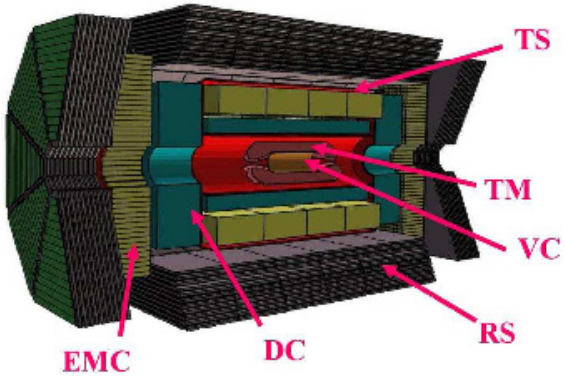

a

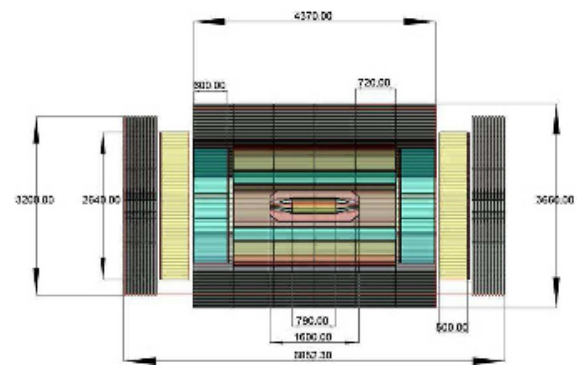

b

Fig. 2. The views of SPD: a) the main parts, b) sizes parts.

consists of "shashlyk" modules with the application of new readout techniques based on avalanche multi pixel diode technology. The expected energy resolution can be $\sigma(E) / E=(5-8) \% / E$. The EMC can be used also for the triggering of MMT-DY electrons. The system of mini-drift layers with Fe layers, called Range System (RS). It can provide a clean (> 99\%) muon identification for muons with momenta greater than $1 \mathrm{GeV}$. The combination of responses from the EMC and the RS can be used for the identfication of pions and protons in a wide energy range. The final version of the SPD will be defined after detailed Monte-Carlo studies.

\section{Simulation of MMT-DY processes}

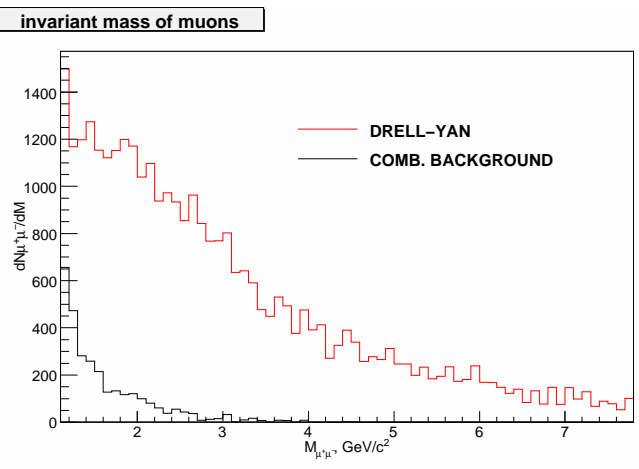

a

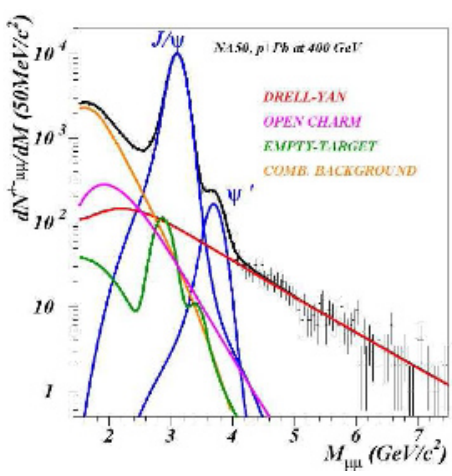

b

Fig. 3. The invariant mass distribution of muon pairs: a) simulation, b) results of COMPASS experiment.

The proton-proton collisions are generated with the PYTHIA 8.1 package implemented on ROOT. Whenever not explicitly stated, all physical processes regarding particles passing through the detector material, are accounted for in the simulation. The interaction point was fixed at the origin of the coordinate system in order to maximize the detector acceptance in particular for the background events. Two data samples are generated (see fig. 3): MMT-DY sample: it contains $1.5 \cdot 10^{6}$ pure MMT-DY events into muon pairs; Background sample: it consists about $3 \cdot 10^{5}$ combinatorial background events.

Preliminary background studies show the good possibility to measure MMT-DY and J/ $\psi$ events with proposed detector.

\section{References}

1. SPD Conceptual Design Report, v.2.1, June 2010, Dubna 